\title{
GCU
}

Glasgow Caledonian

University

University for the Common Good

\section{Reliability analysis of a 3-unit subsystem of a cable plant}

Taj, Syed Zegham; Rizwan, Syed ; Alkali, Babakalli; Harrison, David; Taneja, Gulshan

Published in:

Advances and Applications in Statistics

DOI:

10.17654/AS052060413

Publication date:

2018

Document Version

Publisher's PDF, also known as Version of record

Link to publication in ResearchOnline

Citation for published version (Harvard):

Taj, SZ, Rizwan, S, Alkali, B, Harrison, D \& Taneja, G 2018, 'Reliability analysis of a 3-unit subsystem of a cable plant', Advances and Applications in Statistics, vol. 52, no. 6, pp. 413-429.

https://doi.org/10.17654/AS052060413

\section{General rights}

Copyright and moral rights for the publications made accessible in the public portal are retained by the authors and/or other copyright owners and it is a condition of accessing publications that users recognise and abide by the legal requirements associated with these rights.

Take down policy

If you believe that this document breaches copyright please view our takedown policy at https://edshare.gcu.ac.uk/id/eprint/5179 for details of how to contact us. 


\title{
RELIABILITY ANALYSIS OF A 3-UNIT SUBSYSTEM OF A CABLE PLANT
}

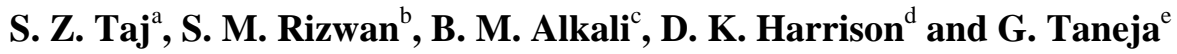 \\ a,b Department of Mathematics and Statistics \\ Caledonian College of Engineering \\ Oman \\ c,d Department of Mechanical Engineering \\ Glasgow Caledonian University \\ Scotland, U. K. \\ ${ }^{\mathrm{e}}$ Department of Mathematics \\ Maharshi Dayanand University \\ India
}

\begin{abstract}
This paper deals with the reliability analysis of a 3-unit subsystem of a cable plant. To facilitate the analysis, ten years maintenance data of the subsystem is collected and the states transition table for the subsystem is developed. Reliability indices of the subsystem such as mean time to failure, availability, expected number of repairs and expected busy period of the repairman are estimated using semiMarkov processes and regenerative point techniques. Simulation is carried out to demonstrate the effect of varying failure/repair rates on the subsystem reliability.
\end{abstract}

Received: April 16, 2018; Accepted: April 25, 2018

2010 Mathematics Subject Classification: 60K10.

Keywords and phrases: reliability, regenerative point technique, semi-Markov process, simulation. 
414 S. Z. Taj, S. M. Rizwan, B. M. Alkali, D. K. Harrison and G. Taneja

\section{Notations}

\begin{tabular}{|c|c|}
\hline MIPM & minor preventive maintenance \\
\hline MAPM & major preventive maintenance \\
\hline MTSF & mean time to subsystem failure \\
\hline$S_{i}$ & state $i$ \\
\hline$g(t)$ & probability density function of repair times \\
\hline$h(t)$ & probability density function of MIPM times \\
\hline$f(t)$ & probability density function of MAPM times \\
\hline$\lambda$ & estimated value of failure rate \\
\hline$\eta$ & estimated value of repair rate \\
\hline$\alpha$ & estimated value of rate of requirement of MIPM \\
\hline$\rho$ & estimated value of rate of performing MIPM \\
\hline$\beta$ & estimated value of rate of requirement of MAPM \\
\hline$\sigma$ & estimated value of rate of performing MAPM \\
\hline$Q_{i j}$ & cumulative distribution function from $S_{i}$ to $S_{j}$ \\
\hline$q_{i j}$ & probability density function from $S_{i}$ to $S_{j}$ \\
\hline (C) & Laplace convolution \\
\hline (S) & Laplace Stieltje’s convolution \\
\hline * & Laplace transform \\
\hline$* *$ & Laplace Stieltje’s transform \\
\hline$A_{0}$ & availability of the subsystem \\
\hline$R_{0}$ & number of subsystem repairs \\
\hline$B_{0}$ & busy period of the repairman \\
\hline
\end{tabular}




\section{Introduction}

Reliability analysis of various industrial systems operating under different conditions and assumptions has been widely discussed by a number of researchers (Taneja et al. [19], Gopalan and Bhanu [3], Tuteja et al. [20], Rizwan et al. [11, 12]). Mathew et al. [6, 7] analyzed a continuous casting plant and studied the variations under different operating conditions. Detailed analysis was reported for a desalination plant by Rizwan et al. [13]. The methodology was further extended for analysis of various industrial systems by Gupta and Gupta [4] with post inspection concept; Ram et al. [10] with waiting repair strategy; Malhotra and Taneja [5] with both units operative on demand. Rizwan et al. [14, 15] then focused on waste water treatment plant and anaerobic batch reactor where reliability indices of interest were obtained in order to assess the plant/reactor performance. Later, extensive system analysis was carried out by Niwas et al. [9] for a single-unit system; Bhardwaj et al. [2] for a redundant system; Adlakha et al. [1] for a two-unit cold standby system; Naithani et al. [8] for a 3-unit induced draft fan system. Rahbi et al. [21] performed reliability analysis of a rodding anode plant in aluminum industry. Taj et al. [16, 17] analyzed two different single machine subsystems of a cable plant with various maintenance categories. Recently, Taj et al. [18] analyzed a subsystem of a cable plant with two machines operating in parallel and priority to repair over preventive maintenance. Hence, the methodology for system analysis has been widely presented in reliability literature. However, analysis of a subsystem (Taj et al. [16, 17, 18]) does not completely contribute to the plant effectiveness in terms of overall performance; it only gives the subsystem effectiveness, and therefore, opens up a scope of complete plant performance as a case study.

Thus, this paper presents reliability analysis of a 3-unit subsystem of an electrical cables manufacturing plant currently operational in Oman. To facilitate the analysis, ten years maintenance data of the subsystem is collected. The data depicts three types of maintenance practices for the subsystem: repair, minor preventive maintenances (MIPM) and major preventive maintenances (MAPM). Repair is carried out upon failure, 
416 S. Z. Taj, S. M. Rizwan, B. M. Alkali, D. K. Harrison and G. Taneja

whereas MIPM/MAPM is performed as per schedule. Priority is given to repair over MAPM. Transition states of the subsystem are shown in Table 1. Detailed subsystem analysis is carried out using semi-Markov processes and regenerative point techniques. Reliability indices of the subsystem namely mean time to failure (MTSF), availability, expected number of repairs and expected busy period of the repairman are estimated. Simulation is also carried out to demonstrate the effect of varying failure/repair rates on the subsystem reliability.

\section{Model Description}

Following operating conditions and assumptions are considered:

- Subsystem consists of three units.

- Each unit undergoes three types of maintenances: repair, MIPM and MAPM.

- Repair is carried out upon failure.

- MIPM/MAPM is carried out as per schedule.

- Priority is given to repair over MAPM.

- During MIPM of one unit, other unit/s do not fail.

- During MAPM of one unit, other unit/s may fail.

- Failure rates are taken as exponential.

- Repair rates are taken as arbitrary.

Possible transition states of the subsystem are described below:

State $0\left(S_{0}\right)$ : all the three units are operating.

State $1\left(S_{1}\right)$ : two units are operating, one unit is under repair.

State $2\left(S_{2}\right)$ : two units are operating, one unit is under MAPM.

State $3\left(S_{3}\right)$ : two units are operating, one unit is under MIPM. 
State $4\left(S_{4}\right)$ : one unit is operating, one unit is under repair, one unit is waiting for repair.

State $5\left(S_{5}\right)$ : one unit is under repair, two units are waiting for repair.

State $6\left(S_{6}\right)$ : one unit is operating, one unit is under repair, one unit is waiting for repair.

State $7\left(S_{7}\right)$ : one unit is operating, one unit is under repair, one unit is waiting for MAPM.

State $8\left(S_{8}\right)$ : one unit is under repair, one unit is waiting for repair, one unit is waiting for MAPM.

$S_{0}, S_{1}, S_{2}, S_{3}, S_{6}$ and $S_{7}$ are regenerative states. $S_{4}, S_{5}$ and $S_{8}$ are nonregenerative states. $S_{4}$ is a failed state. Rates of transition from $S_{i}$ to $S_{j}$ are given in Table 1.

Table 1. Rates of transition for the subsystem

\begin{tabular}{|c|c|c|c|c|c|c|c|c|c|}
\hline$S_{S_{i}} S_{j}$ & $S_{0}$ & $S_{1}$ & $S_{2}$ & $S_{3}$ & $S_{4}$ & $S_{5}$ & $S_{6}$ & $S_{7}$ & $S_{8}$ \\
\hline$S_{0}$ & 0 & $3 \lambda$ & $\beta$ & $\alpha$ & 0 & 0 & 0 & 0 & 0 \\
\hline$S_{1}$ & $g(t)$ & 0 & 0 & 0 & $2 \lambda$ & 0 & 0 & 0 & 0 \\
\hline$S_{2}$ & $f(t)$ & 0 & 0 & 0 & 0 & 0 & 0 & $2 \lambda$ & 0 \\
\hline$S_{3}$ & $h(t)$ & 0 & 0 & 0 & 0 & 0 & 0 & 0 & 0 \\
\hline$S_{4}$ & 0 & $g(t)$ & 0 & 0 & 0 & $\lambda$ & 0 & 0 & 0 \\
\hline$S_{5}$ & 0 & 0 & 0 & 0 & 0 & 0 & $g(t)$ & 0 & 0 \\
\hline$S_{6}$ & 0 & $g(t)$ & 0 & 0 & 0 & 0 & 0 & 0 & 0 \\
\hline$S_{7}$ & 0 & 0 & $g(t)$ & 0 & 0 & 0 & 0 & 0 & $\lambda$ \\
\hline$S_{8}$ & 0 & 0 & 0 & 0 & 0 & 0 & 0 & $g(t)$ & 0 \\
\hline \multicolumn{10}{|c|}{ For non-regenerative states $\left(S_{4}, S_{5}\right.$ and $\left.S_{8}\right)$} \\
\hline \multicolumn{3}{|c|}{$S_{1}$ to $S_{1}$ via $S_{4}$} & \multicolumn{7}{|c|}{$d Q_{11}^{4}(t)=\left(2 \lambda e^{-2 \lambda t} \odot e^{-\lambda t}\right) g(t) d t$} \\
\hline \multicolumn{3}{|c|}{$S_{1}$ to $S_{5}$ via $S_{4}$} & \multicolumn{7}{|c|}{$d Q_{15}^{4}(t)=\left(2 \lambda e^{-2 \lambda t} \odot \lambda e^{-\lambda t}\right) \bar{G}(t) d t$} \\
\hline \multicolumn{3}{|c|}{$S_{7}$ to $S_{7}$ via $S_{8}$} & \multicolumn{7}{|c|}{$d Q_{77}^{8}(t)=\left(\lambda e^{-\lambda t} \odot 1\right) g(t) d t$} \\
\hline \multicolumn{3}{|c|}{$S_{1}$ to $S_{6}$ via $S_{4}$ and $S_{5}$} & \multicolumn{7}{|c|}{$d Q_{16}^{45}(t)=\left(2 \lambda e^{-2 \lambda t} \odot \lambda e^{-\lambda t} \odot 1\right) g(t) d t$} \\
\hline
\end{tabular}


418 S. Z. Taj, S. M. Rizwan, B. M. Alkali, D. K. Harrison and G. Taneja

0 stands for no transition to the mentioned state. Estimated values of various rates for the subsystem are given in Table 2 .

Table 2. Estimated values of rates for the subsystem

\begin{tabular}{|c|l|c|}
\hline S. No. & \multicolumn{1}{|c|}{ Rate (per hour) } & Estimated value (per hour) \\
\hline 1 & $\alpha$, rate of requirement of MIPM & 0.00089 \\
\hline 2 & $\rho$, rate of performing MIPM & 0.88189 \\
\hline 3 & $\beta$, rate of requirement of MAPM & 0.00067 \\
\hline 4 & $\sigma$, rate of performing MAPM & 0.04649 \\
\hline 5 & $\lambda$, failure rate & 0.00336 \\
\hline 6 & $\eta$, repair rate & 0.18908 \\
\hline
\end{tabular}

\section{Transition Probabilities and Mean Sojourn Times}

Transition probabilities from $S_{i}$ to $S_{j}$ are given by the following equations:

$$
\begin{aligned}
& d Q_{01}(t)=3 \lambda e^{(-3 \lambda+\alpha+\beta) t} d t, \\
& d Q_{02}(t)=\beta e^{-(3 \lambda+\alpha+\beta) t} d t, \\
& d Q_{03}(t)=\alpha e^{-(3 \lambda+\alpha+\beta) t} d t, \\
& d Q_{10}(t)=e^{-2 \lambda t} g(t) d t, \\
& d Q_{11}^{4}(t)=\left(2 \lambda e^{-2 \lambda t} @ \lambda e^{-\lambda t}\right) g(t) d t, \\
& d Q_{15}^{4}(t)=\left(2 \lambda e^{-2 \lambda t} @ \lambda e^{-\lambda t}\right) \bar{G}(t) d t, \\
& d Q_{16}^{45}(t)=\left(2 \lambda e^{-2 \lambda t} \odot \lambda e^{-\lambda t} \odot 1\right) g(t) d t, \\
& d Q_{20}(t)=e^{-2 \lambda t} f(t) d t, \\
& d Q_{27}(t)=2 \lambda e^{-2 \lambda t} \bar{F}(t) d t, \\
& d Q_{30}(t)=h(t) d t,
\end{aligned}
$$




$$
\begin{aligned}
& d Q_{61}(t)=g(t) d t, \\
& d Q_{72}(t)=e^{-\lambda t} g(t) d t, \\
& d Q_{77}^{8}(t)=\left(\lambda e^{-\lambda t}(1) g(t) d t .\right.
\end{aligned}
$$

The nonzero elements $p_{i j}$ can be obtained as follows:

$$
\begin{aligned}
& p_{i j}=\lim _{s \rightarrow 0} q_{i j}^{*}(s), \\
& p_{01}=\frac{3 \lambda}{3 \lambda+\alpha+\beta}, \\
& p_{02}=\frac{\beta}{3 \lambda+\alpha+\beta}, \\
& p_{03}=\frac{\alpha}{3 \lambda+\alpha+\beta}, \\
& p_{10}=g^{*}(2 \lambda), \\
& p_{11}^{4}=2 g^{*}(\lambda)-2 g^{*}(2 \lambda), \\
& p_{15}^{4}=1-2 g^{*}(\lambda)+g^{*}(2 \lambda), \\
& p_{16}^{45}=1-2 g^{*}(\lambda)+g^{*}(2 \lambda), \\
& p_{20}=f^{*}(2 \lambda), \\
& p_{27}=1-f^{*}(2 \lambda), \\
& p_{30}=h^{*}(0), \\
& p_{61}=g^{*}(0), \\
& p_{77}^{8}=g^{*}(\lambda),
\end{aligned}
$$


420 S. Z. Taj, S. M. Rizwan, B. M. Alkali, D. K. Harrison and G. Taneja

Following relations can easily be verified:

$$
\begin{aligned}
& p_{01}+p_{02}+p_{03}=1, \\
& p_{10}+p_{11}^{4}+p_{15}^{4}=1, \\
& p_{10}+p_{11}^{4}+p_{16}^{45}=1, \\
& p_{20}+p_{27}=1, \\
& p_{30}=1, \\
& p_{61}=1, \\
& p_{72}+p_{77}^{8}=1 .
\end{aligned}
$$

The mean sojourn time $\mu_{i}$ in the regenerative state $i$ is defined as the time of stay in that state before transition to any other state. If $T$ denotes the sojourn time in the regenerative state $i$, then

$$
\begin{aligned}
& \mu_{i}=E(T)=\int_{0}^{\infty} \operatorname{Pr}[T>t] d t, \\
& \mu_{0}=\frac{1}{3 \lambda+\alpha+\beta}, \\
& \mu_{1}=\frac{1-g^{*}(2 \lambda)}{2 \lambda}, \\
& \mu_{2}=\frac{1-f^{*}(2 \lambda)}{2 \lambda}, \\
& \mu_{3}=\int_{0}^{\infty} \bar{H}(t) d t, \\
& \mu_{6}=\int_{0}^{\infty} \bar{G}(t) d t, \\
& \mu_{7}=\frac{1-g^{*}(\lambda)}{\lambda} .
\end{aligned}
$$


The unconditional mean time $m_{i j}$ taken by the system to transit to any of the regenerative state $j$ when time is counted from the epoch of entry into state $i$, is mathematically stated as

$$
m_{i j}=-\lim _{s \rightarrow 0} q_{i j}^{*^{\prime}}(s)
$$

Following relations can easily be verified:

$$
\begin{aligned}
& m_{01}+m_{02}+m_{03}=\mu_{0}, \\
& m_{10}+m_{11}^{4}+m_{15}^{4}=2 \mu_{7}-\mu_{1}, \\
& m_{10}+m_{11}^{4}+m_{16}^{45}=\mu_{6}, \\
& m_{20}+m_{27}=\mu_{2}, \\
& m_{30}=\mu_{3}, \\
& m_{61}=\mu_{6}, \\
& m_{72}+m_{77}^{8}=\mu_{6} .
\end{aligned}
$$

\section{Reliability Analysis}

\subsection{MTSF}

Let $\phi_{i}(t)$ be the cumulative distribution function of the first passage time from regenerative state $i$ to a failed state $j$. Using probabilistic arguments, the following recursive relations for $\phi_{i}(t)$ are obtained:

$$
\begin{aligned}
& \phi_{0}(t)=Q_{01}(t) \text { (s) } \phi_{1}(t)+Q_{02}(t) \text { s } \phi_{2}(t)+Q_{03}(t) \text { s } \phi_{3}(t), \\
& \phi_{1}(t)=Q_{10}(t) \Subset \phi_{0}(t)+Q_{11}^{4}(t) \text { (s) } \phi_{1}(t)+Q_{15}^{4}(t), \\
& \phi_{2}(t)=Q_{20}(t) \Subset \phi_{0}(t)+Q_{27}(t) \Subset \phi_{7}(t) \text {, } \\
& \phi_{3}(t)=Q_{30}(t) \subseteq \phi_{0}(t),
\end{aligned}
$$


422 S. Z. Taj, S. M. Rizwan, B. M. Alkali, D. K. Harrison and G. Taneja

$$
\begin{aligned}
& \phi_{6}(t)=Q_{61}(t) \Subset \phi_{1}(t), \\
& \phi_{7}(t)=Q_{72}(t) \Subset \phi_{2}(t)+Q_{77}^{8}(t) \Subset \phi_{7}(t) .
\end{aligned}
$$

Taking Laplace Stieltjes transform of above relations and solving for $\phi_{0}^{* *}(s)$, we get

$$
\phi_{0}^{* *}(s)=\frac{N(s)}{D(s)} .
$$

MTSF when the subsystem started at the beginning of state 0 is given by

$$
M T S F=\lim _{s \rightarrow 0} \frac{1-\phi_{0}^{* *}(s)}{s}=\frac{N}{D},
$$

where

$$
\begin{aligned}
N= & \left(1-p_{11}^{4}\right)\left\{p_{72} p_{20}\left(\mu_{0}+p_{03} \mu_{3}\right)+p_{02}\left(p_{72} \mu_{2}+p_{27} \mu_{6}\right)\right\} \\
& -p_{72} p_{20} p_{01}\left(\mu_{1}-2 \mu_{7}\right), \\
D= & p_{72} p_{20} p_{01} p_{15}^{4} .
\end{aligned}
$$

\subsection{Availability of the subsystem}

Using probabilistic arguments of pointwise availability and defining $A_{i}(t)$ as the probability that the subsystem is in up state at instance $t$, given that it enters the regenerative state $i$ at $t=0$, the following recursive relations are obtained:

$$
\begin{aligned}
& A_{0}(t)=M_{0}(t)+q_{01}(t) \Subset A_{1}(t)+q_{02}(t) \Subset A_{2}(t)+q_{03}(t) \Subset A_{3}(t), \\
& A_{1}(t)=q_{10}(t) \Subset A_{0}(t)+q_{11}^{4}(t) \Subset A_{1}(t)+q_{16}^{45}(t) \Subset A_{6}(t), \\
& A_{2}(t)=q_{20}(t) \Subset A_{0}(t)+q_{27}(t) \Subset A_{7}(t), \\
& A_{3}(t)=q_{30}(t) \Subset A_{0}(t), \\
& A_{6}(t)=q_{61}(t) \subseteq A_{1}(t),
\end{aligned}
$$




$$
A_{7}(t)=q_{72}(t) \Subset A_{2}(t)+q_{77}^{8}(t) \Subset A_{7}(t),
$$

here $M_{0}(t)=e^{-(3 \lambda+\alpha+\beta) t}$.

Taking Laplace transform of above equations and solving for $A_{0}^{*}(s)$, we get

$$
A_{0}^{*}(s)=\frac{N_{1}(s)}{D_{1}(s)}
$$

In steady state, availability of the subsystem is given by

$$
A_{0}=\lim _{s \rightarrow 0} s A_{0}^{*}(s)=\frac{N_{1}}{D_{1}}
$$

where

$$
\begin{aligned}
N_{1}= & p_{72} p_{20} p_{10} \mu_{0}, \\
D_{1}= & p_{72} p_{20} p_{10} \mu_{0}+p_{10} p_{02} p_{72} \mu_{2}+p_{72} p_{20} p_{10} p_{03} \mu_{3} \\
& +\left(p_{10} p_{02} p_{27}+p_{72} p_{20} p_{01}+p_{72} p_{20} p_{01} p_{16}^{45}\right) \mu_{6} .
\end{aligned}
$$

\subsection{Expected number of subsystem repairs}

Using probabilistic arguments and defining $R_{i}(t)$ as the expected number of repairs in $(0, t]$, given that the subsystem entered regenerative state $i$ at $t=0$, we get the following recursive relations:

$$
\begin{aligned}
& R_{0}(t)=Q_{01}(t) \subseteq\left\{R_{1}(t)+1\right\}+Q_{02}(t) \subseteq R_{2}(t)+Q_{03}(t) \subseteq R_{3}(t), \\
& R_{1}(t)=Q_{10}(t) \text { (s) } R_{0}(t)+Q_{11}^{4}(t) \subseteq\left\{R_{1}(t)+1\right\}+Q_{16}^{45}(t) \text { (s) }\left\{R_{6}(t)+1\right\} \text {, } \\
& R_{2}(t)=Q_{20}(t) \text { s } R_{0}(t)+Q_{27}(t) \subseteq\left\{R_{7}(t)+1\right\} \text {, } \\
& R_{3}(t)=Q_{30} \text { (s) } R_{0}(t) \text {, } \\
& R_{6}(t)=Q_{61}(t) \subseteq\left\{R_{1}(t)+1\right\} \\
& R_{7}(t)=Q_{72}(t) \text { s } R_{2}(t)+Q_{77}^{8}(t) \subseteq\left\{R_{7}(t)+1\right\} \text {. }
\end{aligned}
$$


424 S. Z. Taj, S. M. Rizwan, B. M. Alkali, D. K. Harrison and G. Taneja

Taking Laplace Stieltjes transform of above equations and solving for $R_{0}^{* *}(s)$, we get

$$
R_{0}^{* *}(s)=\frac{N_{3}(s)}{D_{1}(s)}
$$

In steady state, expected number of repairs per unit time is given by

$$
R_{0}=\lim _{s \rightarrow 0} s R_{0}^{* *}(s)=\frac{N_{3}}{D_{1}}
$$

where $N_{3}=p_{10} p_{02} p_{27}+p_{72} p_{20} p_{01}\left(1+p_{16}^{45}\right), D_{1}$ is already specified

\subsection{Expected busy period of the repairman}

Using probabilistic arguments and defining $B_{i}(t)$ as the probability that the repairman is busy at instance $t$, given that the subsystem entered regenerative state $i$ at $t=0$, we get the following recursive relations:

$$
\begin{aligned}
& B_{0}(t)=q_{01}(t) \Subset B_{1}(t)+q_{02}(t) \Subset B_{2}(t)+q_{03}(t) \Subset B_{3}(t), \\
& B_{1}(t)=W_{1}(t)+q_{10}(t) \odot B_{0}(t)+q_{11}^{4}(t) \odot B_{1}(t)+q_{16}^{45}(t) \odot B_{6}(t), \\
& B_{2}(t)=q_{20}(t) \Subset B_{0}(t)+q_{27}(t) \Subset B_{7}(t), \\
& B_{3}(t)=q_{30}(t) \odot B_{0}(t), \\
& B_{6}(t)=W_{6}(t)+q_{61}(t) \Subset B_{1}(t), \\
& B_{7}(t)=W_{7}(t)+q_{72}(t) \odot B_{2}(t)+q_{77}^{8}(t) \odot B_{7}(t),
\end{aligned}
$$

here $W_{1}(t)=e^{-2 \lambda t} \bar{G}(t), W_{6}(t)=\bar{G}(t), W_{7}(t)=e^{-\lambda t} \bar{G}(t)$.

Taking Laplace transform of above equations and solving for $B_{0}^{*}(s)$, we obtain

$$
B_{0}^{*}(s)=\frac{N_{2}(s)}{D_{1}(s)}
$$


In steady state, expected busy period of the repairman is given by

$$
B_{0}=\lim _{s \rightarrow 0} s B_{0}^{*}(s)=\frac{N_{2}}{D_{1}}
$$

where $N_{2}=p_{10} p_{02} p_{27} \mu_{7}+p_{72} p_{20} p_{01}\left(\mu_{1}+p_{16}^{45} \mu_{6}\right), D_{1}$ is already specified.

\section{Particular Case}

For this particular case, the repair rates are assumed to be exponentially distributed,

$$
\begin{aligned}
& g(t)=\eta e^{-\eta t}, \\
& f(t)=\sigma e^{-\sigma t}, \\
& h(t)=\rho e^{-\rho t} .
\end{aligned}
$$

Using the estimated values from Table 2 and expressions obtained in Section 4, following reliability indices are obtained:

Mean time to subsystem failure $=171342$ hours.

Availability of the subsystem $=0.93951$.

Expected number of subsystem repairs $=0.0099053$.

Expected busy period of the repairman $=0.0090048$.

\section{Simulation}

In this section, the behaviour of subsystem reliability indices viz. MTSF, availability, number of repairs and busy period is studied with $\eta$ and $\lambda$. Table 3 gives the trends of MTSF, availability, number of repairs and busy period for varying values of $\eta$ w.r.t. $\lambda$. 
426 S. Z. Taj, S. M. Rizwan, B. M. Alkali, D. K. Harrison and G. Taneja

Table 3. Effect of $\eta$ on reliability indices w.r.t. $\lambda$

\begin{tabular}{|l|c|c|c|c|}
\hline Reliability index & $\eta=0.1$ & $\eta=0.18908$ & $\eta=0.2$ & $\lambda$ \\
\hline \multirow{4}{*}{ MTSF } & 70786 & 238867 & 266316 & 0.003 \\
\cline { 2 - 5 } & 51135 & 171342 & 190949 & 0.00336 \\
\cline { 2 - 5 } & 31118 & 102965 & 114661 & 0.004 \\
\hline Availability & 0.89889 & 0.94502 & 0.94793 & 0.003 \\
\cline { 2 - 5 } & 0.88898 & 0.93951 & 0.94271 & 0.00336 \\
\cline { 2 - 5 } & 0.87158 & 0.92978 & 0.93348 & 0.004 \\
\hline Number of repairs & 0.0086696 & 0.0088623 & 0.0088743 & 0.003 \\
\cline { 2 - 5 } & 0.0096717 & 0.0099053 & 0.0099199 & 0.00336 \\
\cline { 2 - 5 } & 0.0114324 & 0.0117487 & 0.0117684 & 0.004 \\
\hline Busy period & 0.0082272 & 0.0079332 & 0.0079179 & 0.003 \\
\cline { 2 - 5 } & 0.0093382 & 0.0090048 & 0.0089883 & 0.00336 \\
\cline { 2 - 5 } & 0.0113229 & 0.0109102 & 0.0108916 & 0.004 \\
\hline
\end{tabular}

Following observations are evident from Table 3:

- MTSF decreases w.r.t. $\lambda$ irrespective of $\eta$. The trend reverses with increase in the value of $\eta$, for fixed $\lambda$.

- Availability decreases w.r.t. $\lambda$ irrespective of $\eta$. The trend reverses with increase in the value of $\eta$, for fixed $\lambda$.

- Number of repairs increases w.r.t. $\lambda$ irrespective of $\eta$. The trend remains same with increase in the value of $\eta$, for fixed $\lambda$.

- Busy period increases w.r.t. $\lambda$ irrespective of $\eta$. The trend reverses with increase in the value of $\eta$, for fixed $\lambda$.

\section{Conclusion}

Reliability indices viz. MTSF, availability, expected number of repairs and expected busy period of repairman have been estimated by analysing a 3-unit cable plant subsystem using semi-Markov processes and regenerative point techniques. Subsystem maintenance practices and priority to repair over preventive maintenance have been considered while carrying out the 
analysis. Simulation has been performed to demonstrate the effect of varying failure/repair rates on the subsystem reliability. As a future direction, the analysis could be extended to systems having four or more units, wherein possibility of standby and online maintenance could be considered.

\section{References}

[1] N. Adlakha, G. Taneja and Shilpi, Reliability and cost-benefit analysis of a twounit cold standby system used for communication through satellite with assembling and activation time, Inter. J. Appl. Engin Res. 12(20) (2017), 9697-9702.

[2] R. K. Bhardwaj, Komaldeep Kaur and S. C. Malik, Reliability indices of a redundant system with standby failure and arbitrary distribution for repair and replacement times, Inter. J. System Assurance Engineering and Management 8(2) (2017), 423-431.

[3] M. N. Gopalan and K. S. Bhanu, Cost analysis of a two unit repairable system subject to online preventive maintenance and/or repair, Microelectr. Reliab. 35(2) (1995), 251-258.

[4] S. Gupta and S. K. Gupta, Stochastic analysis of a reliability model of one-unit system with post inspection, post repair, preventive maintenance and replacement, Inter. J. Mechanical Engineering and Robotics Research 2(2) (2013), 178-188.

[5] R. Malhotra and G. Taneja, Stochastic analysis of a two-unit cold stand by system wherein both units may become operative depending upon the demand, J. Qual. Reliab. Engin. 2014 (2014), Art. ID 896379, 13 pp. http://dx.doi.org/10.1155/2014/896379.

[6] A. G. Mathew, S. M. Rizwan, M. C. Majumder and K. P. Ramachandran, Reliability modelling and analysis of a two unit continuous casting plant, J. Franklin Institute 348(7) (2011), 1488-1505.

[7] A. G. Mathew, S. M. Rizwan, M. C. Majumder, K. P. Ramachandran and G. Taneja, Reliability analysis of identical two-unit parallel cc plant system operative with full installed capacity, Inter. J. Perform. Engin. 7(2) (2011), 179-185.

[8] A. Naithani, B. Parashar, P. K. Bhatia and G. Taneja, Probabilistic analysis of a 3-unit induced draft fan system with one warm standby with priority to repair of the unit in working state, Inter. J. System Assurance Engineering and Management 8 (2017), 1383-1391. https://doi.org/10.1007/s13198-017-0608-6. 
[9] R. Niwas, M. S. Kadyan and J. Kumar, MTSF (mean time to system failure) and profit analysis of a single-unit system with inspection for feasibility of repair beyond warranty, Inter. J. System Assurance Engineering and Management 7(1) (2016), 198-204.

[10] M. Ram, S. B. Singh and V. V. Singh, Stochastic analysis of a standby system with waiting repair strategy, IEEE Trans. Systems, Man and Cybernetics: Systems 43(3) (2013), 698-707.

[11] S. M. Rizwan, V. Khurana and G. Taneja, Modelling and optimization of a singleunit plc system, Inter. J. Model. Simul. 27(4) (2007), 361-368.

[12] S. M. Rizwan, V. Khurana and G. Taneja, Reliability analysis of a hot standby industrial system, Inter. J. Model. Simul. 30(3) (2010), 315-322.

[13] S. M. Rizwan, N. Padmavathi, A. Pal and G. Taneja, Reliability analysis of a seven unit desalination plant with shutdown during winter season and repair/ maintenance on FCFS basis, Inter. J. Perform. Engin. 9(5) (2013), 523-528.

[14] S. M. Rizwan, J. V. Thanikal, N. Padmavathi and H. Yazidi, Reliability and availability analysis of an anaerobic batch reactor treating fruit and vegetable waste, Inter. J. Appl. Engin. Res. 10(24) (2015), 44075-44079.

[15] S. M. Rizwan, J. V. Thanikal and M. Torrijos, A general model for reliability analysis of a domestic waste water treatment plant, Inter. J. Condition Monitoring and Diagnostic Engineering Management 17(3) (2014), 3-6.

[16] S. Z. Taj, S. M. Rizwan, B. M. Alkali, D. K. Harrison and G. Taneja, Reliability modelling and analysis of a single machine subsystem of a cable plant, 7th International Conference on Modelling, Simulation and Applied Optimization, American University of Sharjah, Sharjah, UAE, 2017. https://doi.org/10.1109/ICMSAO.2017.7934917.

[17] S. Z. Taj, S. M. Rizwan, B. M. Alkali, D. K. Harrison and G. L. Taneja, Reliability analysis of a single machine subsystem of a cable plant with six maintenance categories, Inter. J. Appl. Engin. Res. 12(8) (2017), 1752-1757.

[18] S. Z. Taj, S. M. Rizwan, B. M. Alkali, D. K. Harrison and G. Taneja, Probabilistic modelling and analysis of a cable plant subsystem with priority to repair over preventive maintenance, i-manager’s J. Math. 6(3) (2017), 12-21.

[19] G. Taneja, S. M. Rizwan and R. Kumar, Profit analysis of a system with perfect repair at partial failure and priority for repair to complete failed unit, Pure Appl. Math. Sci. 35(1/2) (1992), 131-141. 
[20] R. K. Tuteja, S. M. Rizwan and G. Taneja, Profit evaluation of a two unit cold standby system with tiredness and two types of repairman, J. Indian Soc. Stat. Oper. Res. 21(1-4) (2000), 1-10.

[21] Yaqoob Al Rahbi, S. M. Rizwan, B. M. Alkali, Andrew Cowell and G. Taneja, Reliability analysis of rodding anode plant in aluminium industry, Inter. J. Appl. Engin. Res. 12(16) (2017), 5616-5623. 